\title{
Lidil
}

Revue de linguistique et de didactique des langues

$44 \mid 2011$

Langues, minor(is)ations et marginalisations

\section{Assimilation linguistique et processus de minoration}

Maria Zerva

\section{OpenEdition}

\section{Journals}

Édition électronique

URL : http://journals.openedition.org/lidil/3147

DOI : 10.4000/lidil.3147

ISSN : 1960-6052

Éditeur

UGA Éditions/Université Grenoble Alpes

\section{Édition imprimée}

Date de publication : 15 décembre 2011

Pagination : 125-136

ISBN : 978-2-84310-212-7

ISSN : $1146-6480$

Référence électronique

Maria Zerva, "Assimilation linguistique et PRocessus de minoration », Lidil [En ligne], 44 | 2011 mis en ligne le 15 juin 2013, consulté le 01 mai 2019. URL : http://journals.openedition.org/lidil/3147 ; DOI : 10.4000/lidil.3147 


\title{
Assimilation linguistique et processus de minoration ${ }^{1}$
}

\author{
Maria Zerva*
}

\begin{abstract}
RÉSUMÉ
Dans cet article, nous abordons le phénomène de l'assimilation linguistique et, plus particulièrement, les facteurs qui sont susceptibles de le favoriser ou, au contraire, d'endiguer sa progression. Nous prenons appui sur les facteurs développés par Holmes (1992), que nous discutons à la lumière d'un cas précis, celui des descendants de réfugiés grecs orthodoxes turcophones d'Asie Mineure, installés en Grèce au milieu des années 1920.
\end{abstract}

\begin{abstract}
Language shift is a widely studied phenomenon, whose progress may be encouraged or delayed (or even cancelled) by contextual factors. In this paper, we will examine the factors suggested by Holmes (1992) in the light of a particular case. Our discussion will focus on evidence from a sociolinguistic survey of Greek orthodox descendants of Asia Minor refugees, who resettled in Greece in the mid-1920s.
\end{abstract}

\section{Quelques considérations théoriques}

Le concept de contact des langues est omniprésent dans la littérature sociolinguistique de ces dernières décennies et le fait que tout individu et groupe soit constamment confronté à plusieurs variétés ou langues semble être aujourd'hui reconnu comme un phénomène ordinaire. Néanmoins, on constate que le contact entre des langues amène très fréquemment l'individu ou le groupe à opérer un choix au profit d'une langue, entrainant l'abandon de l'autre. Le plus souvent, la langue finalement

* Université de Strasbourg.

1. Je tiens à remercier Irini Tsamadou-Jacoberger et Cécile Jahan pour leur relecture attentive de cet article et leurs commentaires constructifs. 
retenue est «l'intrus » (langue B), tandis que celle d'origine (langue A) est graduellement délaissée.

Ainsi peut-on décrire schématiquement le phénomène de l'assimilation linguistique (language shift), phénomène qui se trouve au cœur de nombreuses études sociolinguistiques, à l'instar de celles de Susan Gal (1979), Lukas Tsitsipis (1998), Joshua Fishman (1991) ou encore Fishman et al. (1985). Pour une vue d'ensemble des questions que soulève ce phénomène, on se réfèrera à Matthias Brenzinger (1997), ainsi qu'aux chapitres qui s'y rapportent dans des ouvrages introductifs à la sociolinguistique, comme ceux de Janet Holmes (1992), Peter Trudgill (2000), Florian Coulmas (2005) ou encore Nelli Kostoula-Makraki (2001).

Tous ces travaux montrent que l'assimilation ne se produit pas soudainement mais graduellement. Avant l'abandon final de la langue d'origine (langue $\mathrm{A}$ ) au profit de la langue $\mathrm{B}$, une restriction fonctionnelle de la langue A s'opère (Tsitsipis, 1998 : 2-3; voir le functional shift de Brenzinger, 1997 : 277), généralement sur trois générations, comme dans le cas, largement étudié, des migrants.

Plusieurs facteurs sont associés au phénomène en question : démographiques, politiques, économiques, socioculturels, institutionnels ou symboliques, comme par exemple l'image ou le prestige des langues A et B. Ces facteurs favorisent ou freinent l'assimilation linguistique. À la complexité de la combinaison entre l'ensemble des facteurs présents ou absents et le caractère local de la situation s'ajoutent les attitudes des locuteurs (voir le concept de language loyalty, Brenzinger 1997 : 277), puisque l'abandon d'une langue relèverait finalement d'une décision, plus ou moins libre, prise à la fois individuellement et collectivement.

Il serait insensé de vouloir prétendre à une grille de facteurs « universelle », qui conviendrait à toute situation d'assimilation linguistique. Dans cet article, nous discuterons les facteurs qui, selon Janet Holmes (1992 : 65-73 et notamment 71-73), contribuent à la résistance contre l'assimilation linguistique et favorisent ainsi le maintien de la langue $\mathrm{A}^{2}$. Il s'agit des facteurs suivants :

1. Démographiques et sociaux. Plus précisément, le maintien de la langue A est favorisé dans des environnements ruraux et isolés, quand les membres de la communauté habitent les uns près des autres et se voient fréquemment, ou encore quand les mariages

2. Dans le texte de Holmes (1992), les différents points ne sont pas présentés sous forme de liste, mais la grille est le produit de notre résumé des pages 65-73. 
mixtes sont découragés et que l'organisation familiale encourage l'usage de la langue A (ex. la famille élargie habite sous le même toit). Enfin, la taille du groupe semble également être un facteur important en faveur du maintien de la langue A.

2. Institutionnels. L'usage et le soutien de la langue A dans des domaines comme l'éducation, l'administration, la justice, la religion et les médias peuvent être cruciaux pour son maintien.

3. Contacts avec la patrie. «Le degré et la fréquence des contacts avec la patrie » $(1992: 71)$ contribuent également au maintien de la langue A. De nouveaux migrants ou simplement des visiteurs sont autant de raisons et d'occasions qui permettent de continuer à parler la langue $\mathrm{A}$, tout comme la perspective pour les migrants ou leurs enfants d'aller s'installer au pays d'origine ou simplement de le visiter. Il s'agit en effet d'une « orientation positive envers la patrie », comme cela est précisé ailleurs par Holmes et al. (2001 : 60 et 65-66). Cela ne concerne pas que la première génération, mais aussi celles qui lui succèdent.

4. Attitudes et valeurs. La langue B exerce de fortes pressions sur les locuteurs de la langue A, en ce qu'elle est porteuse de promesses en termes d'insertion et d'ascension sociales pour les personnes qui choisiraient l'assimilation linguistique. Pour y résister, les valeurs dont la langue A est investie jouent un rôle très important, puisqu'elles conditionnent les attitudes de ses locuteurs : le fait que la langue A constitue un symbole identitaire pour le groupe en question, que les locuteurs soient fiers de leur langue, de leur identité ethnique et de leur culture d'origine, sont autant d'éléments qui encouragent le soutien au maintien de la langue A.

C'est à partir de cette grille que nous aborderons la question de l'assimilation linguistique dans deux communautés, présentées ci-après.

\section{Terrain d'étude}

Le présent article prend appui sur une étude sociolinguistique de deux communautés villageoises grecques, où deux langues ont été, et sont toujours, en contact depuis près d'un siècle : le grec et le turc. Il s'agit des villages de Bafra (situé au nord-ouest du pays, à $8 \mathrm{~km}$ d'Ioannina) et de Nea Bafra (au nord-est, à $49 \mathrm{~km}$ de Serrès et à $24 \mathrm{~km}$ de Drama). Les deux villages ont été fondés dans les années 1920 pour loger des réfugiés 
d'Asie Mineure, dans le cadre de l'échange obligatoire de populations entre la Grèce et la Turquie. Cet échange, prévu en 1923 par la Convention de Lausanne, mettait ainsi un terme au conflit gréco-turc des années précédentes. Le critère qui a déterminé le déplacement forcé a été de nature religieuse. Le critère religieux résultait de l'ancienne distinction des peuples dans l'Empire ottoman en millets, qui ne tenait pas compte de l'appartenance ethnique. De fait, tous les chrétiens orthodoxes d'Asie Mineure (à quelques exceptions près, environ 1,5 million ${ }^{3}$ ) ont pris la route pour la Grèce, de même que la quasi-totalité des musulmans de Grèce (environ 350000 ) a dû partir en Turquie. Les réfugiés acquéraient immédiatement la nationalité du pays d'accueil et étaient interdits de retour au pays natal.

Parmi les 1,2 million de réfugiés recensés en Grèce en 1928, quelques années après l'échange, environ 100000 étaient turcophones, dont les premiers habitants de Bafra et Nea Bafra. En 2005 et 2007, nous nous sommes rendue dans ces communautés, où nous avons effectué 36 entretiens semi-directifs (23 à Bafra et 13 à Nea Bafra, correspondant respectivement à 540 et 382,5 minutes d'enregistrement ${ }^{4}$ ). Nos interlocuteurs appartenaient aux deuxième, troisième et quatrième générations de réfugiés, et ceux relevant de la première génération avaient plus de 12 ans au moment du déplacement.

Le passage d'un monolinguisme général en turc de la première génération, à un bilinguisme (turc et grec) ou à un monolinguisme en grec (ou un bilinguisme entre le grec et une langue autre que le turc) pour les générations suivantes, amène à considérer que le phénomène d'assimilation linguistique est en cours dans les communautés. Or, l'analyse du corpus fait ressortir que 29 témoins sur $36\left(80,55 \%{ }^{5}\right)$ déclarent avoir une maitrise solide du turc (maitrise qui rentre dans les catégories très bien, bien ou moyennement bien), tandis que 5 sur $36(13,9 \%)$ le maitri-

3. Sur les chiffres, voir Ladas, 1932; Hirschon, 2003 et 1998.

4. Ce corpus, ainsi que la méthodologie adoptée pour son recueil, sont présentés en détail dans notre recherche, menée dans le cadre de notre thèse de doctorat (Zerva, 2011).

5. Nous établirons des pourcentages malgré le petit nombre des témoins, parce que nous pensons que, de cette façon, nous serons plus à même de suivre les dynamiques sociolinguistiques au sein des deux communautés. Certes, le nombre de personnes interrogées ne nous permet pas de le considérer comme représentatif de l'ensemble des habitants des deux villages, mais il reste pour autant significatif de la situation. 
seraient peu et seulement 2 sur 36 (5,55\%) pas du tout. Par conséquent, une première remarque qui peut être faite est que la langue $\mathrm{A}$, le turc, résiste très bien aux pressions exercées par la langue $\mathrm{B}$, le grec, et cela bien au-delà des trois générations que la littérature sociolinguistique évoque comme période d'accomplissement de l'assimilation.

Il convient de voir à présent comment la maitrise du turc se répartit selon les différentes générations et, partant, comment évolue l'assimilation linguistique dans le temps (Gal, 1979 : 100-101). Ainsi, nous constatons que tous les locuteurs de la deuxième génération maitrisent très bien le turc $(100 \%)$, tandis que ce pourcentage diminue au fil des générations : $75 \%$ pour la troisième et seulement $25 \%$ pour la quatrième. Si on raisonne en termes de maitrise solide, où on regrouperait les catégories très bonne maitrise, bonne et moyenne, les pourcentages se présentent ainsi : $100 \%$ pour la deuxième génération, $87,5 \%$ pour la troisième et $37,5 \%$ pour la quatrième. Le tableau qui suit donne une vue d'ensemble de l'évolution des compétences au fil des générations.

\begin{tabular}{|c|c|c|c|c|c|c|c|c|c|c|c|}
\hline \multicolumn{2}{|c|}{ Total (36) } & $\begin{array}{c}\text { Très bien/ } \\
\text { Bien }\end{array}$ & $\begin{array}{c}\text { Bien / Moyenne- } \\
\text { ment bien }\end{array}$ & \multicolumn{2}{c|}{$\begin{array}{c}\text { Moyenne- } \\
\text { ment bien }\end{array}$} & \multicolumn{2}{|c|}{ Peu } & \multicolumn{2}{c|}{$\begin{array}{c}\text { Pas du } \\
\text { tout }\end{array}$} \\
\hline $2^{\text {e }}$ génér. & 12 & 12 & $100 \%$ & 0 & $0 \%$ & 0 & $0 \%$ & 0 & $0 \%$ & 0 & $0 \%$ \\
\hline $3^{\text {e }}$ génér. & 16 & 12 & $75 \%$ & 1 & $6,25 \%$ & 1 & $6,25 \%$ & 2 & $12,5 \%$ & 0 & $0 \%$ \\
\hline $4^{\text {e }}$ génér. & 8 & 2 & $25 \%$ & 1 & $12,5 \%$ & 0 & $0 \%$ & 3 & $37,5 \%$ & 2 & $25 \%$ \\
\hline
\end{tabular}

Tableau 1. - Maitrise à Bafra et Nea Bafra du turc selon les générations.

Ce tableau fait apparaitre la perte du turc au fil des générations et montre que l'assimilation linguistique, si elle n'est pas accomplie au bout de quatre générations, le sera probablement avec la cinquième. $\mathrm{Si}$, en outre, on prend en considération également la variable âge, il ressort que les personnes de la quatrième génération qui ont une très bonne maitrise du turc sont nées avant 1970. Ainsi nous constatons que ceux qui sont nés à partir du début ou du milieu des années 1970 ont une maitrise faible ou nulle du turc, à une exception près. Cela témoigne du refus de transmettre le turc aux nouvelles générations, refus apparu aux alentours de la fin de la seconde guerre mondiale et généralisé après $1970^{6}$.

6. Si on compare les deux villages, la transition vers le grec s'est faite une décennie plus tôt à Nea Bafra qu'à Bafra (environ 1965 vs. env. 1975). Ces 
Ce qui nous intéressera, dans ce qui suit, est d'examiner si et comment les différents critères de la grille que nous avons établie à partir de Janet Holmes se présentent dans le cas des Grecs orthodoxes turcophones. Pour ce faire, nous développerons, selon les besoins, les spécificités des communautés et quelques résultats de l'analyse de notre corpus d'entretiens. Il ne s'agira pas de faire des prédictions concernant l'avenir de l'assimilation linguistique, mais plutôt de fournir des éléments d'interprétation sur la dynamique actuelle.

\section{Confrontation de la grille de Holmes avec le corpus}

Nous commencerons par le deuxième facteur retenu par Janet Holmes, à savoir le facteur institutionnel, parce qu'il permettra d'examiner davantage certains aspects du contexte politico-historique dans lequel ces populations ont évolué. Ainsi, à la question de savoir si le turc était présent dans des domaines institutionnels, la réponse est assez simple : non. Le turc n'est nullement présent ni reconnu de façon officielle dans le cas des réfugiés orthodoxes d'Asie Mineure ou de leurs descendants ${ }^{7}$.

En ce qui concerne plus particulièrement le domaine de la religion, le turc n'y trouve pas non plus de place; la possibilité d'utiliser le turc dans les offices orthodoxes étant hors de propos aujourd'hui et même envisagé comme un sacrilège. Même dans le contexte de l'Empire ottoman, il n'est pas sûr que le grec ait été remplacé par le turc dans les offices religieux ${ }^{8}$ et s'il l'a été, on ignore à quel degré. Si nous insistons sur le domaine de la religion, c'est que la religion a servi d'ancrage identitaire fondamental pour ces populations, faute de l'autre élément crucial de l'identité grecque, à savoir la langue grecque ${ }^{9}$.

remarques s'appuient sur les analyses menées dans le cadre de notre thèse, ouvr. cité, voir notamment chapitres 3.2. et 3.3.

7. Le turc est par ailleurs reconnu comme langue de la minorité musulmane, seule minorité qui a une existence officielle aux yeux de l'État grec. Cette minorité musulmane, protégée par le Traité de Lausanne de 1923, est la seule qui bénéficie de mesures réglementaires qui assurent à sa langue (le turc) une reconnaissance officielle dans différents domaines institutionnels. Néanmoins, il faut souligner que, dans le contexte grec, les turcophones musulmans et orthodoxes ne sont en aucun cas assimilables.

8. Sur le sujet, voir Clogg (2004 : 341-355 et $1999: 119-122)$.

9. En effet, l'identité grecque, telle qu'elle est conçue par l'idéologie dominante et véhiculée par les discours nationalistes, s'articule autour de deux pôles : 
Ainsi, nous constatons l'absence de reconnaissance institutionnelle du turc dans le cas des Grecs orthodoxes turcophones et, qui plus est, selon nos enquêtes, l'absence totale de la part de la population de toute volonté de revendiquer une place pour le turc dans ces domaines. Les Grecs orthodoxes turcophones ont en effet intégré les discours nationalistes dominants - qui exigeaient leur hellénisation complète - et ont renoncé sans résistance, semble-t-il, à leur langue d'origine. On peut alors les considérer comme un groupe minoré. Nous entendons la minoration comme une dévalorisation qualitative et quantitative ${ }^{10} \mathrm{~d}$ 'un groupe humain à travers sa langue (Blanchet, 2000 : 131).

Pour ce qui est du troisième facteur, le fait que ces populations aient été déplacées de façon obligatoire et irrévocable suite à une décision politique a exclu tout contact (ou presque) avec la patrie : il n'y a pas eu de nouvelle vague de réfugiés qui serait venue de la Turquie en Grèce, puisque la totalité de la population a quitté le pays natal. Parallèlement il n’y avait aucune perspective de retour, puisque celui-ci était banni par la Convention de Lausanne ${ }^{11}$.

La possibilité de s'entretenir en turc avec des personnes extérieures à la communauté arrive sporadiquement; sauf lorsque des Turcs d'Allemagne, en rentrant en Turquie en voiture, passent par Bafra ou, lors d'une visite du lieu d'origine en Turquie, sorte de pèlerinage dans les terres natales des parents ou grands-parents généralement effectué par les deuxième et troisième générations, qui d'ailleurs maitrisent le turc. Enfin, le contact avec le turc se produit grâce aux antennes satellites qui permettent de regarder la télévision turque. Cependant, tout cela reste

l'orthodoxie et la langue grecque (qui sert de lien direct avec l'Antiquité classique, autre élément fondamental de l'identité nationale grecque). Sur le sujet, voir Zerva (2010).

10. «Réduction numérique proportionnelle ou brute des locuteurs par annexion dans un groupe alloglotte plus nombreux et/ou diminution du nombre d'occurrences et de locuteurs de la langue en question à l'intérieur de son groupe originel, ce qui signifie qu'une partie des membres du groupe est amenée à adopter la langue dominante majoritaire, ponctuellement ou largement » (Blanchet, 2000 : 131). Philippe Blanchet appelle minorisation le volet quantitatif et ailleurs (Blanchet, $2005: 34$ ) minoritarisation l'ensemble des deux processus. Nous opterons pour le seul terme minoration pour des raisons de commodité.

11. Convention concernant l'échange des populations grecques et turques, et protocole, qui a été signée le 30 janvier 1923 à Lausanne et a précédé la signature du Traité de Lausanne (24 juillet 1923). 
extrêmement marginal dans la vie des communautés et ne concerne que les personnes qui parlent déjà le turc, ne constituant pas une motivation pour l'apprendre. En effet, les orthodoxes turcophones ne s'identifient pas aux Turcs.

En revanche, on constate une orientation positive envers la patrie pour les réfugiés turcophones et leurs descendants (les deuxième et troisième générations incluses). Cette orientation relève, dans les discours, d'un « contact » imaginaire : la terre natale, l'Asie Mineure, est idéalisée dans l'imaginaire collectif des réfugiés, mais aussi de tous les Grecs. Ainsi, l'image d'une Asie Mineure-paradis hellénique s'est développée, sans l'élément turc ou seulement en toile de fond ${ }^{12}$. Dans ce contexte, malgré un contact intense avec la patrie; la langue turque n'a pas été valorisée, en raison d'une hellénisation rétrospective - et imaginaire des lieux.

Le quatrième facteur - attitudes et valeurs - constitue, à nos yeux, la clé de compréhension du processus d'assimilation linguistique tel qu'il apparait chez les Grecs orthodoxes turcophones. Il semble en effet que le choix identitaire de ce groupe, à savoir l'identification aux Grecs et à leur idée nationale, ait conditionné le sort de la langue turque ${ }^{13}$.

Ayant fait ce choix, il fallait prendre ses distances par rapport à la langue turque, langue de l'Autre national par excellence (Grec $\neq$ Turc). Cela signifiait abandonner l'usage du turc, ou, plutôt, dire l'abandonner, parce que malgré un fort engagement dans le discours, une très grande partie de nos interlocuteurs maitrise effectivement le turc ${ }^{14}$, comme nous avons pu le voir (voir tableau 1 et commentaires). L'abandon du turc serait considéré comme un retour à l'« état naturel » relaté par un récit historico-mythique qui circule largement au sein des communautés : à un

12. Voir notamment Balta et Papataxiarchis (2002 : 42-47). Sur l'idéalisation de 1'Asie Mineure dans la littérature, voir Mackridge (2003 : 243-244).

13. On ne sait pas vraiment ce que les turcophones orthodoxes pensaient d'eux dans l'Empire ottoman, à savoir entre le $\mathrm{XV}^{\mathrm{e}}$ siècle et 1923. L'identification des populations au sein de l'Empire était fondée sur l'attachement religieux et local, au sens restreint du terme (le village d'origine). Toutefois, toute ambiguïté ou incertitude concernant l'identité des turcophones orthodoxes a été résolue de facto avec l'échange des populations. Or, nous parlons ici de choix identitaire parce que leur intégration dans la nation grecque n'a en aucun cas été forcée mais, en revanche, a été revendiquée.

14. Il faut toutefois souligner que le plus jeune de nos interlocuteurs avait 22 ans en 2005. Ainsi ne savons-nous pas comment se présente la turcophonie chez les plus jeunes membres des communautés. 
moment donné dans l'Empire ottoman, les ancêtres des Turcs auraient été contraints de choisir entre leur religion (christianisme orthodoxe) et leur langue (naturellement, le grec). La légende raconte qu'ils auraient choisi de sauvegarder la religion et seraient ainsi devenus turcophones.

Cela expliquerait que le turc ne constitue pas un symbole de leur identité. Si les Turcs se déclarent fiers de leur identité et de leur culture d'origine, celles-ci apparaissent dissociées de la langue turque et étroitement liées au monde hellénique, à l'image de la construction imaginaire d'une Asie Mineure hellénisée. Leur identification se résume brièvement comme Grec, d'origine d'Asie Mineure, ou Grec, d'origine réfugiée.

La prise de distance d'avec le turc, qui concrètement se traduit par l'abandon et la non-transmission du turc, notamment à partir du milieu des années 1970, se manifeste également sur le plan discursif. Ainsi, dans les entretiens, on peut relever que des traces de minoration du turc sont systématiquement repérées dans l'agencement du discours : choix lexicaux, ordre des mots, marqueurs de qualification et de quantification, modalité ${ }^{15}$, commentaires, mais aussi recours à l'implicite, marques de gêne à propos du turc ou stratégies de justification et d'argumentation : la minoration du turc, en tant que langue, se construit à la fois par rapport au turc standard, mais aussi par rapport à la variante locale des témoins turc local ${ }^{16}$. Celle-ci est associée à des évaluatifs axiologiques négatifs tels que $\chi \circ v \tau \varrho o \varkappa o \mu \mu \varepsilon ́ v \alpha$ (grossier) ou encore $\chi \omega \varrho \iota d ́ \tau \iota \varkappa \alpha$ (paysan). Les locuteurs minorent aussi systématiquement leurs compétences en turc ou son importance dans les pratiques et le répertoire des communautés. Ainsi on voit se dessiner une représentation du turc comme langue extérieure au locuteur et au groupe, extérieure à l'identité personnelle et collective.

15. Un exemple caractéristique est l'association du turc à la modalité déontique à travers l'adverbe $\alpha v \alpha \gamma \varkappa \alpha \sigma \tau \iota x \alpha$ (obligatoirement) : j'ai/ nous avons obligatoirement appris le turc. Nous considérons cette association comme indice de minoration et d'extériorité du turc à l'essence identitaire des personnes.

16. Un autre volet intéressant de la représentation du turc est l'effort fréquent de dissocier leur turc local du turc standard en insistant sur les différences, l'absence d'intercompréhension, qui va jusqu'à dire que leur langue n'est pas du turc, mais un mélange de grec pontique, de turc et d'autres langues, inventé pour tromper les Ottomans, une astuce pour leur faire croire qu'ils avaient obéi face au dilemme posé et qu'ils avaient effectivement abandonné leur langue (le grec). 
En revanche, la mise en discours du grec est toute différente. Celui-ci est posé, seul ou renforcé dans sa valeur assertive par des adjectifs affirmatifs ou une intonation ascendante ${ }^{17}$. Le grec apparait ainsi toujours majoré dans le discours de nos interlocuteurs, jamais négocié.

Il est remarquable que cette minoration et cette extériorité de la langue turque soient instrumentalisées dans le processus de la construction identitaire ${ }^{18}$ de nos interlocuteurs. Le but est de se construire une identité grecque et, pour ce faire, la minoration et la prise de distance avec le turc s'avèrent particulièrement utiles.

Reste à discuter pourquoi le turc a tout de même plutôt bien résisté à la pression exercée par le grec, et ce jusqu'à la troisième et même la quatrième génération. Une explication possible relèverait du premier facteur de notre grille, à savoir celui de nature sociodémographique. Ainsi, le fait de vivre dans des villages habités uniquement par des turcophones, majoritairement des agriculteurs, au moins lors des quatre ou cinq premières décennies, a sans doute joué un rôle important dans le maintien du turc. Les mariages mixtes étaient par ailleurs rares pour la deuxième génération et peu fréquents pour la troisième. Cette situation était donc propice à la pratique du turc jusqu'à la troisième génération incluse.

\section{Conclusion}

Il ressort de cette analyse que, mis à part l'environnement sociodémographique, aucun facteur n'a véritablement œuvré pour le maintien du turc. Le grec dominait de facto tous les aspects du nouvel espace de vie; il était par ailleurs, en tant que langue liturgique, intimement associé à l'orthodoxie, élément essentiel de l'identité de nos interlocuteurs, mais aussi langue de l'identité nationale à laquelle ils ont choisi de s'identifier. Même leur passé dans l'Empire ottoman a été rétrospectivement épuré de tout élément turc. Le turc apparait dénué de contenu symbolique, ce qui aurait entrainé sa minoration systématique (repérable dans le discours) et son abandon. Il nous semble que l'intérêt de la grille de

17. Rising intonation (pitch) : la voix monte jusqu'à une note assez élevée, mais cela ne concerne pas les questions. Cela peut caractériser un mot ou un énoncé, en entier ou en partie.

18. Nous l'entendons non pas comme une construction flambant neuve, ex nihilo, mais comme une construction additive ou une re-confirmation des constructions antérieures. 
Holmes est non seulement de permettre une analyse globale des facteurs contingents qui influent sur l'avancement ou pas de l'assimilation linguistique, mais aussi d'appréhender les facteurs plus symboliques, liés aux valeurs et aux attitudes, individuelles ou collectives.

\section{RÉFÉRENCES BIBLIOGRAPHIQUES}

Balta E. et Papataxiarchis A. (2002) : « Dealing with Cultural Difference: 'Asia Minor Refugee' and 'Muslim Minority' Folkore Studies in Greece », dans VI. Milletlerarasi Türk Halk Kültürü Kongresi. Son Elli Yılda Türkiye Dışındaki Türk Halk Kültürü Çalışmaları Seksiyon Bildirileri, Ankara, p. 38-53. Disponible sur <http://evangeliabalta. com/?catid=books4> [consulté le 07/06/2009].

Blanchet P. (2000) : La linguistique de terrain. Méthode et théorie. Une approche ethno-sociolinguistique, Rennes, Presses universitaires de Rennes. - (2005) : «Minorations, minorisations, minorités : Essai de théorisation d'un processus complexe », dans D. Huck et P. Blanchet (dir.) : Minorations, minorisations, minorités. Etudes exploratoires. Cahiers de sociolinguistique $n^{\circ} 10$, Rennes, Presses universitaires de Rennes, p. 17-47.

BRENZINGER M. (1997) : « Language Contact and Language Displacement », dans F. Coulmas (éd.), The Handbook of Sociolinguistics. Oxford/Massachusetts, Blackwell Publishers, p. 273-284.

ClogG R. (1999) : «A Millet within a Millet: The Karamanlides », dans D. Gondicas et C. Issawi (éds), Ottoman Greeks in the Age of Nationalism. Princeton/New Jersey. The Darwin Press INC, p. 115-142.

- (2004) : « The Publication and Distribution of Karamanli Texts by the British and Foreign Bible Society before $1850 »$, Parties I et II, dans R. Clogg, I kath'imas Anatoli. Studies in Ottoman Greek History, Istanbul. The Isis Press, p. 333-360 et 361-386, publié originellement dans [1968], Journal of Ecclesiastical History XIX, p. 57-81 et 171-193.

Coulmas F. (2005) : Sociolinguistics. The study of speakers' choices, Cambridge University Press.

Fishman J. A. (1991) : Reversing Language Shift. Clevedon/Philadelphia/ Adelaide, Multilingual Matters.

Fishman J. M., Gertner H., Lowy E. et Milán W. (éds) (1985) : The Rise and Fall of the Ethnic Revival: Perspectives on Language and Ethnicity, Berlin/New York/ Amsterdam, Mouton Publishers.

GaL S. (1979) : Language shift. Social determinants of linguistic change in bilingual Austria, Nea York/San Francisco/London, Academic Press.

Hirschon R. (2003) : « The Consequences of the Lausanne Convention. An Overview », dans R. HIRSCHON (éd), Crossing the Aegean. An appraisal 
of the compulsory population exchange between Greece and Turkey. New York/ Oxford. Berghahn Books, p. 13-20.

Hirschon R. (1998/1989) : Heirs of the Greek Catastrophe. The Social Life of Asia Minor Refugees in Piraeus. New York/Oxford, Berghahn Books (Oxford, Clarendon Press pour la première édition).

Holmes J. (1992) : An Introduction to Sociolinguistics, London and New York, Longman.

Holmes J., Roberts M., Verivaki M. et 'Aipolo 'A (2001) : « Language maintenance and shift in three New Zealand speech communities », dans A. Georgakopoulou et M. Spanaki (éds), A Reader in Greek Sociolinguistics, Bern, Peter Lang, p. 41-71 [1 ère édition dans Applied Linguistics (1993), $\mathrm{n}^{\circ}$ 14(1), Oxford University Press].

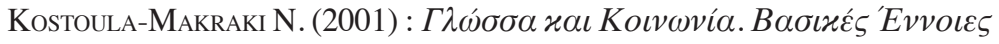
(Langue et Société. Notions de base), Athènes, Metechmio.

LADAs P. (1932) : The exchange of Minorities: Bulgaria, Greece and Turkey, New York, The Macmillan Company.

Mackridge P. (2003) : «The Myth of Asia Minor in Greek Fiction », dans R. Hirschon (éd.), Crossing the Aegean. An appraisal of the compulsory population exchange between Greece and Turkey, New York/Oxford, Berghahn Books, p. 235-246.

TRUdGILl P. (2000) : Sociolinguistics: an introduction to language and society, Londres, Penguin [1 1 ère edition, 1974 chez Pelican Books].

Tsitsipis L. (1998) : A Linguistic Anthropology of Praxis and Language shift. Arvanitika (Albanian) and Greek in Contact, Oxford, Clarendon Press.

Zerva M. (2011) : Les Grecs turcophones orthodoxes : une étude sociolinguistique, thèse de doctorat, université de Strasbourg.

- (2010) : «Le citoyen grec par excellence. Conception normative du citoyen grec par l'État-nation grec et cas de marginalité », dans N. Abi-Rached (dir.), Normes et marginalités à l'épreuve, Strasbourg, Presses universitaires de Strasbourg, p. 49-61. 